\title{
Produção de frutos e distribuição espacial de angiospermas com frutos zoocóricos em uma Floresta Ombrófila Mista no Rio Grande do Sul, Brasil
}

\author{
GABRIELA PAISE ${ }^{1}$ e EMERSON M. VIEIRA ${ }^{1,2}$
}

(recebido: 29 de janeiro de 2003; aceito: 29 de setembro de 2005)

\begin{abstract}
Fruit production and spatial distribution of animal-dispersed angiosperms in a Mixed Ombrophilous Forest in State of Rio Grande do Sul, Brazil). We investigated, during 14 months, patterns of fruit production of animal dispersed angiosperms in a patch of Mixed Ombrophilous Forest (MOF) in the National Park of Aparados da Serra, State of Rio Grande do Sul, south Brazil. For a total of 570 individuals sampled in a 2.56 ha area we identified 27 species of animal dispersed angiosperms (13 families). The most abundant families were: Myrtaceae (eight species), Melastomataceae, Solanaceae, and Rubiaceae (three species each). Most sampled life forms were shrubs (46.0\% of all individuals $37.0 \%$ of species total), followed by treelets (31.9\% and $22.2 \%)$, herbs (10.2\% and $18.5 \%)$, trees (8.7\% and $18.5 \%)$, and epiphytes $(3.2 \%$ and $3.7 \%)$. Red $(22.2 \%)$ and orange (18.5\%) fruits prevailed, as well as small fruits ( $70 \%$ of fruit total with size of $1-10 \mathrm{~mm} \times 1-10 \mathrm{~mm})$, and fruits with one to ten small seeds ( $96 \%$ of total species with seed size of $1-10 \mathrm{~mm} \times 1-10 \mathrm{~mm}$ ). The three species that produced the greatest amount of fruits were: Myrceugenia miersiana (Gardner) D. Legrand \& Kausel (Myrtaceae) (tree $-61.0 \%$ of total production of fruits), and the melastomes Leandra variabilis Cogn. (shrub - 18.1\%) and Miconia cinerascens Miq. (shrub - 8.6\%). These species showed an aggregated dispersion pattern (Standardized Morisita index $>0.5$ for all of them). The monthly number of fruiting species was significantly correlated with monthly temperature in the same month $\left(r_{s}=0,70 ; p<0,01\right)$, and with rainfall in the previous month $\left(r_{s}=0,67 ; p<0,02\right)$. The seed production of Araucaria angustifolia (Bertol.) Kuntze occurred during the period with the lowest number of angiosperm species producing animal-dispersed fruits. This non-overlapping between events might favor the occurrence of frugivorous animals, enabling a higher resource offer during the entire year.
\end{abstract}

Key words - fruit production, Mixed Ombrophilous Forest, phenology, spatial distribution, zoochory

RESUMO - (Produção de frutos e distribuição espacial de angiospermas com frutos zoocóricos em uma Floresta Ombrófila Mista no Rio Grande do Sul, Brasil). Foram investigados os padrões de produção de frutos de angiospermas zoocóricas, ao longo de 14 meses, em Floresta Ombrófila Mista (FOM) no Rio Grande do Sul, Brasil. Para um total de 570 indivíduos, amostrados em uma área de 2,56 ha, foram identificadas 27 espécies de angiospermas zoocóricas (13 famílias). As famílias mais ricas em espécies foram: Myrtaceae (oito espécies), Melastomataceae, Solanaceae e Rubiaceae (três espécies cada). A maioria das plantas amostradas era arbusto (46,0\% do total de indivíduos, 37,0\% do total de espécies), seguido por arvoretas (31,9\% e $22,2 \%)$, ervas (10,2\% e 18,5\%), árvores $(8,7 \%$ e $18,5 \%)$ e epífitas $(3,2 \%$ e $3,7 \%)$. Frutos de cor vermelha (22,2\%) e laranja $(18,5 \%)$ predominaram, assim como frutos pequenos (70\% do total de frutos mediam entre $1-10 \mathrm{~mm} \times 1-10 \mathrm{~mm})$, e com uma a dez sementes pequenas $(96 \%, 1-10 \mathrm{~mm} \times 1-10 \mathrm{~mm})$. As três espécies que mais contribuíram para a produção total de frutos foram: Myrceugenia miersiana (Gardner) D. Legrand \& Kausel (Myrtaceae) (hábito arbóreo - 61,0\% do total) e as melastomatáceas Leandra variabilis Cogn. (arbusto - 18,1\%) e Miconia cinerascens Miq. (arbusto - 8,6\%). Essas espécies apresentaram distribuição espacial agrupada (ìndice de Morisita Padronizado $>0,5$ para todas). O número de espécies produzindo frutos por mês esteve significativamente correlacionado com a temperatura mensal do mesmo mês $\left(r_{s}=0,70 ; p<0,01\right)$ bem como com a precipitação total mensal do mês anterior $\left(\mathrm{r}_{\mathrm{s}}=0,67 ; \mathrm{p}<0,02\right)$. O período de produção de sementes de Araucaria angustifolia (Bertol.) Kuntze ocorreu em uma época em que havia um menor número de angiospermas produzindo frutos zoocóricos. Essa não sobreposição entre eventos pode favorecer a ocorrência de animais frugívoros, possibilitando uma oferta mais constante de recursos durante todo o ano.

Palavras-chave - distribuição espacial, fenologia, Floresta Ombrófila Mista, produção de frutos, zoocoria

\section{Introdução}

A Floresta Ombrófila Mista (FOM) é uma formação florestal típica do Sul do Brasil, ocorrendo

\footnotetext{
1. Universidade do Vale do Rio dos Sinos, Laboratório de Ecologia de Mamíferos, Centro 2, Caixa Postal 275, 93022-000 São Leopoldo, RS, Brasil.

2. Autor para correspondência: emersonmv@unisinos.br
}

nos Estados do Rio Grande do Sul, Santa Catarina e Paraná, nas partes mais altas do Planalto Sul-Brasileiro (Jarenkow \& Baptista 1987) com algumas manchas esparsas em áreas de maior altitude do sudeste do Brasil. A FOM é dominada pela araucária ou pinheiro-doparaná [Araucaria angustifolia (Bertol.) Kuntze], que é a espécie arbórea mais abundante dessa formação vegetal (Reitz \& Klein 1966), sendo também conhecida como floresta com araucária (Fernandes \& Bezerra 
1990). Atualmente, a distribuição da FOM representa cerca de $4 \%$ da sua área original, de cerca de $200.000 \mathrm{~km}^{2}$ (Leite \& Klein 1990). No Rio Grande do Sul, essa formação está sendo constantemente destruída por atividades agrícolas e de pastagens, restando apenas 9.195,65 km² de Florestas Ombrófilas Mistas (Cademartori et al. 2002), ou menos de $50 \%$ da área de $21.213 \mathrm{~km}^{2}$ coberta originalmente (Backes 1999).

Em florestas tropicais são encontradas espécies florescendo e frutificando o ano todo, embora haja diferenças, ao longo dos meses e nas estações, no número de espécies produzindo flores e frutos (Talora \& Morellato 2000). Estas flutuações são freqüentemente relacionadas com fatores endógenos (e.g. genéticos, Alencar 1994) e fatores ambientais, principalmente precipitação e luz (Morellato et al. 2000). No entanto fatores bióticos, como a ação de animais dispersores e predadores de frutos e sementes, são atualmente considerados como fatores seletivos importantes na determinação dos padrões de frutificação das plantas (Morellato \& Leitão Filho 1992). Em florestas tropicais, pelo menos $50 \%$ a $75 \%$ das espécies de árvores produzem frutos carnosos, consumidos por aves ou mamíferos (Howe \& Smallwood 1982).

A produção de sementes do principal componente arbóreo das FOM, a araucária, ocorre no inverno, entre os meses de março e agosto (Reitz et al. 1988, Carvalho 1994, Mantovani et al. 2004). Já para as angiospermas associadas à essas florestas, embora ocorram geralmente entre 25 e 30 espécies em um única área (Klein 1984), há somente um estudo sobre fenologia das espécies (Marques et al. 2004) e nenhum estudo enfocando os modos de dispersão dessas. A possibilidade de haver uma segregação temporal na produção de frutos/sementes de angiospermas dispersas por animais e a araucaria suscita questões ecologicamente relevantes tanto em relação à variação sazonal na oferta de recursos para frugívoros quanto em relação a uma possível competição por dispersores entre os grupos de plantas.

Plantas com frutos zoocóricos apresentam uma série de características que estimulam e facilitam o seu consumo por animais e, potencialmente, a dispersão de suas sementes (Barroso et al. 1999, Mikich \& Silva 2001). A distribuição temporal e espacial desses recursos (frutos e sementes) pode influenciar fortemente a distribuição de animais frugívoros. De maneira similar, o comportamento dos dispersores, e mesmo a composição da guilda local de frugívoros, contribuem para os padrões de distribuição espacial exibidos pelas plantas zoocóricas (Howe 1980, Howe et al. 1985, Howe 1986). Desta forma, o conhecimento das características morfológicas dos frutos, bem como dos padrões de distribuição espacial das plantas zoocóricas, são importantes para a investigação das interações entre essas plantas e seus dispersores em áreas de florestas.

No presente estudo foram investigados os padrões de produção de frutos de angiospermas zoocóricas, ao longo de 14 meses, em um fragmento natural de FOM no sul do Brasil e também a distribuição espacial das principais espécies de angiospermas zoocóricas da área. Esses padrões foram avaliados sob um enfoque de fonte de recursos para vertebrados frugívoros. As principais questões formuladas foram: 1. Quais espécies de angiospermas zoocóricas são responsáveis pela maior produção de frutos em uma FOM e, conseqüentemente, seriam potencialmente as mais importantes como recurso alimentar para os animais da área? 2. Quais as características morfológicas dos frutos zoocóricos em uma área de FOM? 3. Qual a distribuição espacial de espécies de angiospermas zoocóricas nessa área? 4. Qual é a variação sazonal na produção de frutos na área? 5. Qual a relação entre o período de frutificação com o período de produção de sementes de A. angustifolia e com os fatores climáticos (temperatura, precipitação)?

\section{Material e métodos}

Área de estudo - O estudo foi desenvolvido em uma área de Floresta Ombrófila Mista, no Parque Nacional de Aparados da Serra (PNAS), no Rio Grande do Sul, Brasil (29¹0' S e $50^{\circ} 06^{\prime} \mathrm{W}$ ), a uma altitude média de $950 \mathrm{~m}$. O Parque, que possui área de 10.000 ha, é coberto por um mosaico de manchas de floresta com araucária (que cobre cerca de $40 \%$ da área) entremeados aos campos de altitude e aos banhados. A área estudada encontra-se na região subtropical (Rizzini 1992), o clima é do tipo Cfb, segundo a classificação de Köppen (1948), úmido com verões brandos característicos do Planalto das araucárias, no Rio Grande do Sul (Backes et al. 2000). A temperatura média anual é de $14,5^{\circ} \mathrm{C}$, a média das máximas do mês mais quente é de $20,9^{\circ} \mathrm{C}$ e a do mês mais frio é de $5,7^{\circ} \mathrm{C}$ (Backes 1999). A precipitação total anual varia entre 1.750 e 2.250 mm (Estação Metereológica de Cambará do Sul) e é normalmente bem distribuída ao longo do ano (Rambo 1953).

Produção de frutos - Acompanhou-se a frutificação (produção de frutos maduros - modificado de Morellato et al. 1989) das angiospermas zoocóricas entre abril/2000 e maio/2001, em um fragmento de FOM de aproximadamente 25 ha. No interior desse fragmento demarcou-se uma área quadrada de 2,56 ha $(160 \mathrm{~m} \times 160 \mathrm{~m})$ onde, mensalmente, durante cinco a seis dias, foram localizados todos os indivíduos que estavam produzindo frutos zoocóricos maduros. Não foi encontrada, ao longo do estudo, nenhuma 
espécie com frutos não zoocóricos. Entre abril/2000 e setembro/2000 a produção de frutos individual foi estimada como alta (mais de 50\% da copa apresentando a fenofase) ou baixa (menos de $50 \%$ da copa apresentando a fenofase). A partir de outubro/2000, cada indivíduo encontrado foi incluído em uma das cinco categorias de intensidade de Fournier (1974): 0 - ausência da fenofase; $1-1 \%$ a $25 \%$ da copa apresentando a fenofase; $2-26 \%$ a $50 \%$ da copa apresentando a fenofase; $3-51 \%$ a $75 \%$ da copa apresentando a fenofase e $4-76 \%$ a $100 \%$ da copa apresentando a fenofase. Para comparação dos resultados da categorização de Fournier com aqueles obtidos antes de outubro/2000, indivíduos nas categorias de intensidade 1 e 2 foram classificados como de baixa produção e indivíduos nas categorias acima de 2 foram considerados como de alta produção. Com isso foi possível acompanhar a produção mensal de frutos zoocóricos ao longo de todo o período de estudo. Para uma estimativa da produção total de frutos da área, foram somados os valores atribuídos a cada indivíduo para a obtenção de um valor total para a área estudada. Os valores estimados para cada espécie foram obtidos através da soma dos valores atribuídos aos indivíduos da espécie. Para essas estimativas foram usados somente os dados obtidos quando usamos as cinco categorias de intensidade descritas acima. Embora com isso essas estimativas tenham sido baseadas em oito meses, foi durante esse período em que houve produção de frutos (total ou em sua maior parte) de $92,5 \%$ das espécies. Devido a isso, acredita-se que a estimativa obtida foi bastante representativa da produção total e da contribuição das principais espécies zoocóricas para essa produção.

Para investigar qual a relação entre características das espécies e produção de frutos, estas foram caracterizadas de acordo com seu hábito, tipo de fruto, cor do fruto e características morfométricas (tamanho do fruto e das sementes e número de sementes por fruto). Usou-se as categorias de hábitos sugeridas por Reitz et al. (1988) e Backes \& Nardino (2003): árvore, arvoreta, arbusto, erva, epífita. Para tipo e cor de fruto usou-se a nomenclatura sugerida por Barroso et al. (1999) a saber, tipo: aquênio, baga, drupa, fruto múltiplo, melanídio, núcula, solanídio; cor dos frutos maduros: amarelo, azul, laranja, vermelho, verde, violeta, rosa, roxo.

Visando avaliar a distribuição dos recursos disponíveis para os animais foi investigado o padrão espacial das espécies zoocóricas com maior produção de frutos. Para isso, a área de estudo (2,56 ha) foi subdividida em parcelas quadradas $(15 \mathrm{~m} \times 15 \mathrm{~m})$ e foi utilizado o índice de dispersão de Morisita padronizado $\mathrm{I}_{\mathrm{p}}$ (Krebs 1989).

Realizou-se também o registro, na área de estudo, do período de produção de sementes (pinhão) de Araucaria angustifolia (Bertol.) Kuntze, por ser essa a principal fonte potencial de recursos para os animais frugívoros/granívoros da área (Cademartori 1994, Bordignon \& Monteiro Filho 1999). Para testar a hipótese de que haveria um maior número de angiospermas produzindo frutos durante meses com menor oferta de recursos (i.e. sem pinhão) o número médio de espécies que estavam produzindo frutos nos meses em que havia a produção de pinhão foi comparado com os meses em que não havia produção de pinhão por meio do teste de MannWhitney (como em Vieira \& Izar 1999). Para avaliar a correlação entre temperatura e precipitação locais e a produção de frutos utilizou-se o teste de Correlação de Spearman, $\mathrm{r}_{\mathrm{s}}$ (Zar 1996), com o auxílio do programa estatístico SYSTAT 8.0 (Wilkinson 1998). Os dados ambientais foram obtidos da Estação de Metereologia do Parque Nacional de Aparados da Serra. Além da comparação direta entre dados ambientais e produção de frutos testou-se também se havia defasagem entre condições ambientais e respostas fenológicas das espécies, por meio de correlações entre o número de espécies produzindo frutos e os dados ambientais de até dois meses anteriores. As espécies observadas tiveram material coletado e preparado, sendo as exsicatas resultantes depositadas no Herbário Aluísio Sehnem (HASU) do Centro de Ciências da Saúde na Universidade do Vale do Rio dos Sinos (Unisinos). A identificação do material coletado foi realizada com auxílio de taxonomistas e comparação com exsicatas do Herbarium Anchieta - PACA e Herbário Aluísio Sehnem HASU (Unisinos). O sistema de classificação adotado segue o modelo de Cronquist (1988).

\section{Resultados}

Para um total de 570 indivíduos amostrados, foram identificadas 27 espécies de angiospermas zoocóricas pertencentes a 13 famílias, não tendo sido encontrada nenhuma espécie não-zoocórica. As famílias mais ricas em espécies foram: Myrtaceae (oito espécies), Melastomataceae, Solanaceae e Rubiaceae (três espécies cada) (tabela 1). Desse total, a maioria era de arbustos (46,0\% do total de indivíduos, 37,0\% do total de espécies), seguido por arvoretas $(31,9 \%$ e $22,2 \%)$, ervas $(10,2 \%$ e $18,5 \%)$, árvores $(8,7 \%$ e $18,5 \%)$ e epífitas $(3,2 \%$ e $3,7 \%)$.

Embora representassem apenas 31,9\% do total de indivíduos amostrados, os indivíduos da categoria arvoreta $(\mathrm{n}=182)$ foram responsáveis por $49,0 \%$ da produção total estimada de frutos (somatório de todos os valores do índice de Fournier estimados). Já o hábito arbustivo, embora tenha sido a categoria com o maior número de indivíduos ( $\mathrm{n}=262$ ), contribuiu com $34,2 \%$ da produção total de frutos estimada. O hábito herbáceo ( $\mathrm{n}=58$ indivíduos) contribuiu com $5,9 \%$ da produção total, sendo que o hábito arbóreo, com apenas 50 indivíduos, responderam por $8,9 \%$ dessa produção e o hábito epifítico $(n=18)$ apresentou a menor taxa de produção de frutos, com $2,0 \%$ da produção total na área estudada. No ambiente estudado, as seis espécies que 


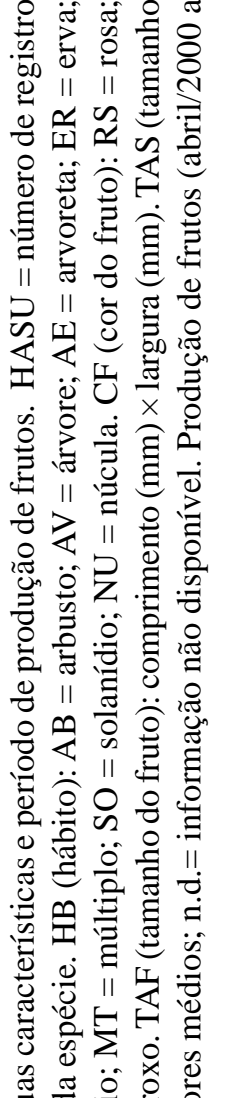

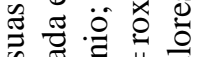

के

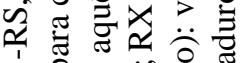

i

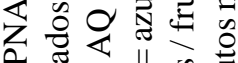

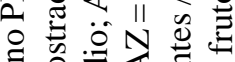

ส뉴워

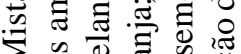

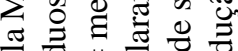

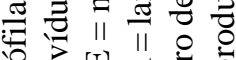

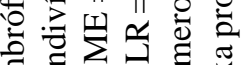

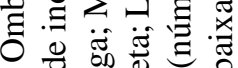

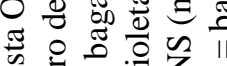

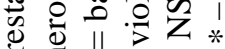

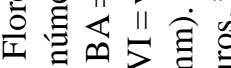

छ II

学司要

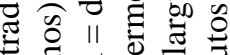

के

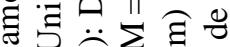

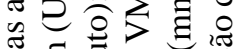

ह

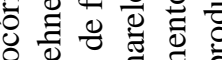

\&

N.융 중

造焉出若

की

등

뜬

青
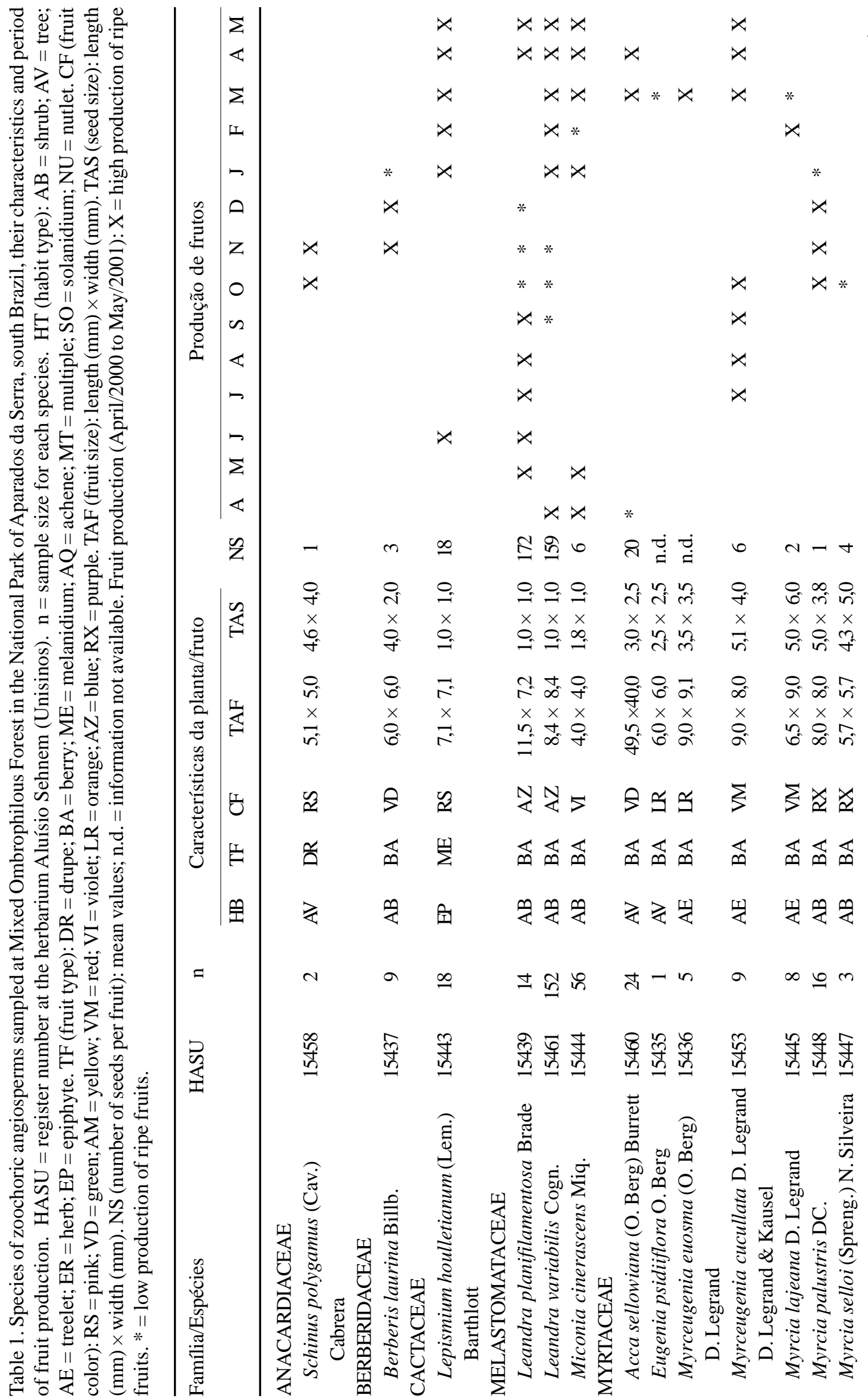


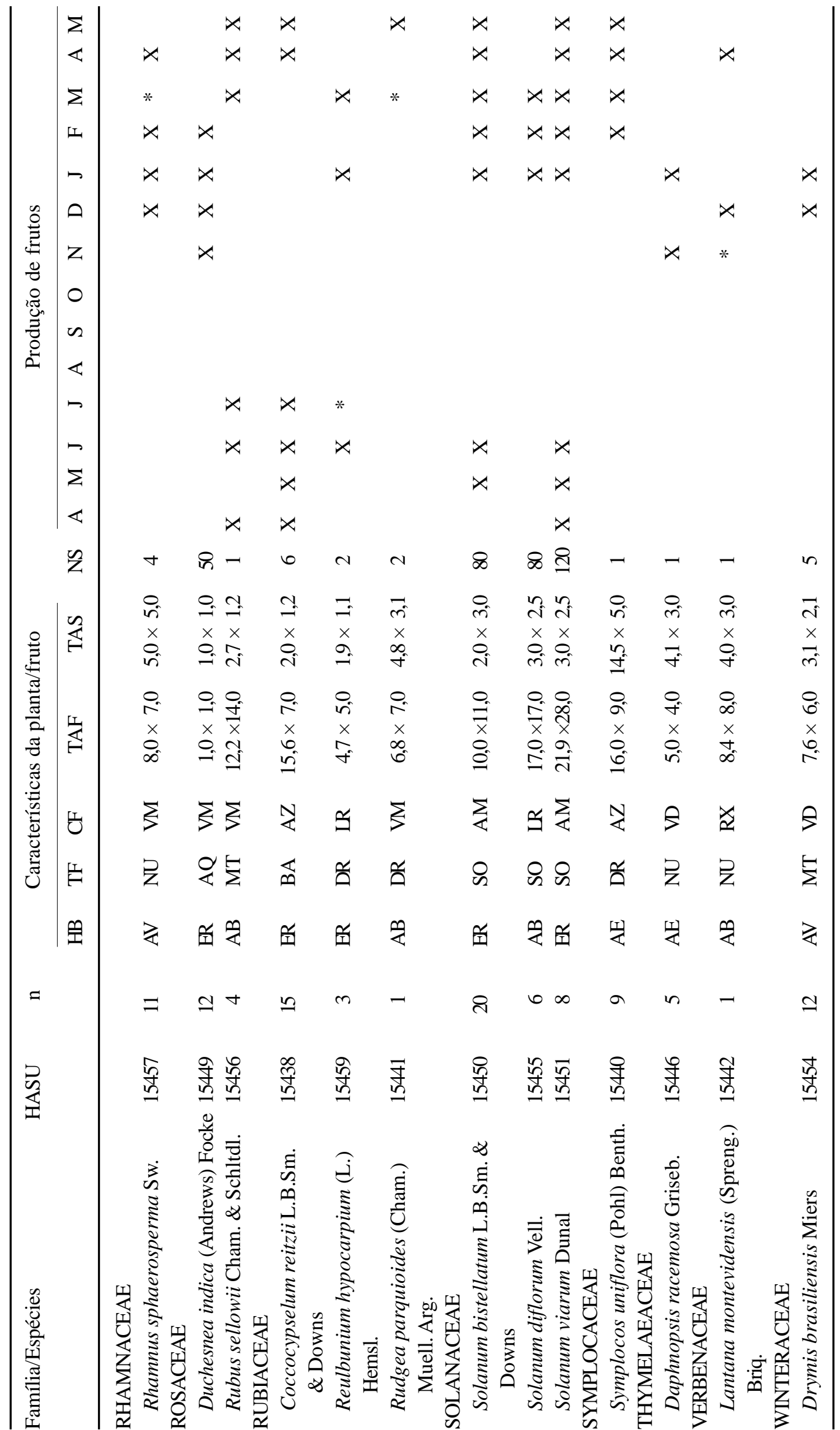


mais contribuíram para a produção total de frutos foram: Myrceugenia miersiana (61,0\% da produção total de frutos estimada), Leandra variabilis $(18,1 \%)$, Miconia cinerascens (8,6\%), Acca selowiana (4,1\%), Solanum bistellatum $(1,6 \%)$ e Leandra planifilamentosa $(1,1 \%)$.

As espécies encontradas apresentaram frutos dos tipos "carnosos indeiscentes" baga ( $\mathrm{n}=13$ espécies), núcula $(\mathrm{n}=4)$, drupa $(\mathrm{n}=3)$, solanídio $(\mathrm{n}=3)$, múltiplo $(n=2)$, melanídio $(n=1)$ e aquênio $(n=1)$. A coloração dos frutos zoocóricos foi variada (oito cores), com a maioria das espécies possuindo frutos vermelho $(n=6)$ e laranja $(n=5)$ (tabela 1$)$.

O tamanho dos frutos variou entre 1 e $40 \mathrm{~mm}$ de largura e 1 a $50 \mathrm{~mm}$ comprimento ( $\mathrm{n}=27$ espécies), com $70 \%$ das espécies apresentando largura entre $1 \mathrm{e}$ $10 \mathrm{~mm}$ e $77 \%$ das espécies com comprimento entre $1 \mathrm{e}$ $10 \mathrm{~mm}$ (tabela 1). As sementes apresentaram tamanho relativamente pequeno, com largura variando entre $1 \mathrm{e}$ $10 \mathrm{~mm}$ e comprimento variando entre 1 e $20 \mathrm{~mm}$ (96\% das espécies com comprimento variando entre $1 \mathrm{e}$ $10 \mathrm{~mm}$ ). O número de sementes variou de 1 a 172 sementes por fruto, mas a maioria $(63 \%)$ das espécies possuía de 1 a 10 sementes (tabela 1). De um total de 27 espécies analisadas, $37 \%$ possuíam apenas uma semente. Espécies cujos frutos possuíam 50 ou mais sementes representaram apenas $22 \%$ do total de espécies.

Dentre as seis espécies com maior número de indivíduos amostrados $(\mathrm{n}=14$ a $\mathrm{n}=152)$ e com os maiores períodos de frutificação (três a 10 meses) (tabela 1), 83,3\% apresentaram padrão espacial significativamente agrupado, como L. variabilis, $\mathrm{I}_{\mathrm{p}}=0,52$ $(\mathrm{p}=0,05) ; M$. cinerascens, $\mathrm{I}_{\mathrm{p}}=0,50(\mathrm{p}<0,01)$; A. sellowiana, $\mathrm{I}_{\mathrm{p}}=0,52(\mathrm{p}<0,01) ;$ M. miersiana, $\mathrm{I}_{\mathrm{p}}$ $=0,50(\mathrm{p}<0,01) ;$ e S. bistellatum, $\mathrm{I}_{\mathrm{p}}=0,51(\mathrm{p}<0,01)$. Somente L. planifilamentosa apresentou padrão espacial aleatório $\left(I_{p}=-0,17, p>0,60\right)$.

Os padrões de frutificação das espécies variaram com o hábito da planta (figura 1). A categoria de hábito cujas espécies com produção de frutos se distribuíram mais uniformemente ao longo de todo o ano foi a dos arbustos (figura 1A). Arvoretas apresentaram frutos ao longo da maior parte do ano, com um pico na estação mais quente e úmida (figura 1B). Já as árvores não frutificaram entre os meses de maio e setembro, no período de produção de pinhões (figura 1C). Quanto às ervas, houve um período com pouca ou nenhuma produção de frutos, de agosto a dezembro (figura 1D). Frutos da única espécie de epífita encontrada foram registrados em maio/2000 e entre janeiro e maio/2001.
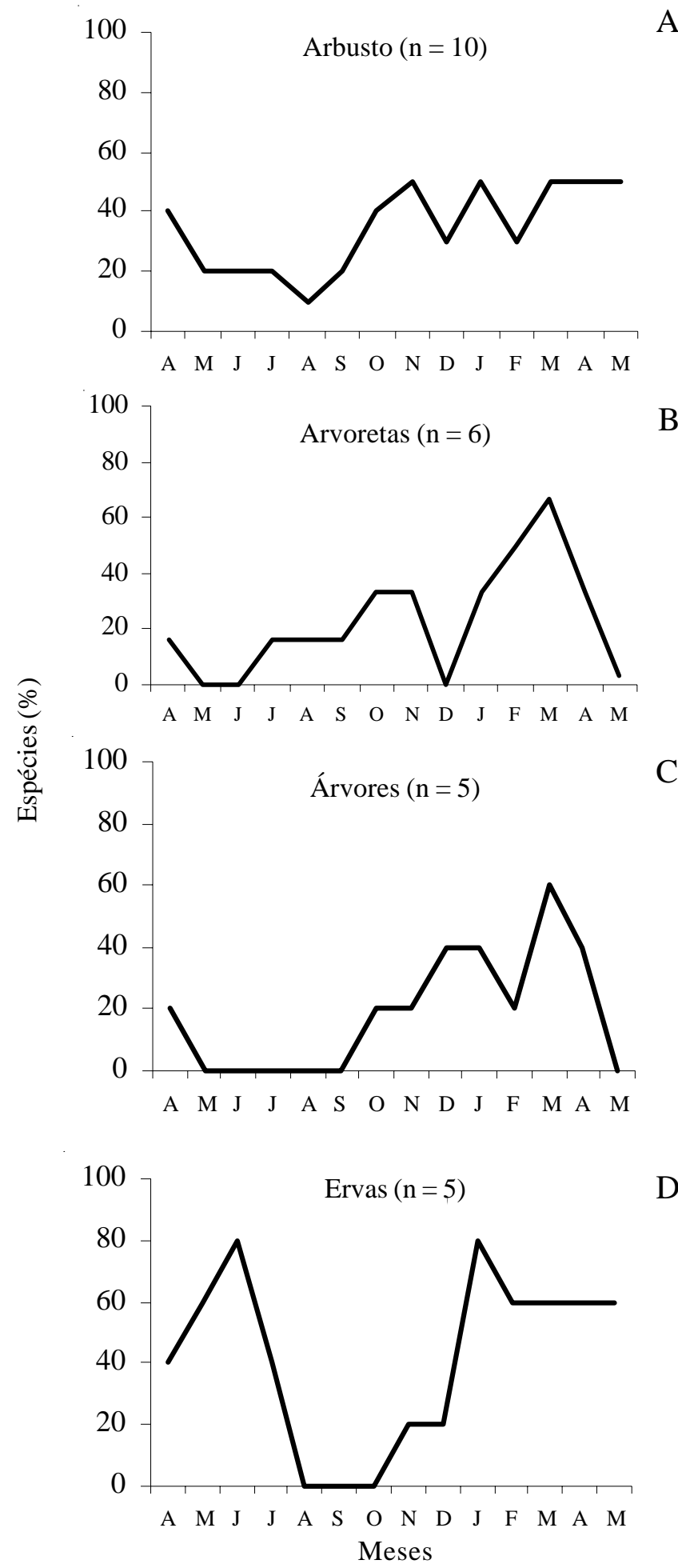

Figura 1. Frutificação de angiospermas zoocóricas em relação ao hábito das espécies (número total de espécies por hábito entre parênteses) entre abril/2000 e maio/2001 em Floresta Ombrófila Mista.

Figure 1. Monthly percentage of zoocoric angiosperms producing fruits according to their habit (total number of species per habit between parenthesis) from April/2000 to May/2001 in a Mixed Ombrophilous Forest in south Brazil. 
Entre os meses de abril a agosto, período de baixas temperaturas e baixa precipitação, houve a produção de sementes de A. angustifolia (pinhão) (G. Paise, dados não publicados), gimnosperma característica da Floresta Ombrófila Mista (figura 2). Nesse período, o número médio mensal de espécies de angiospermas produzindo frutos (média $=6,8 ;$ d.p. $=3,8$ ) foi menor do que o número médio mensal de espécies produzindo frutos durante os meses em que não havia produção do
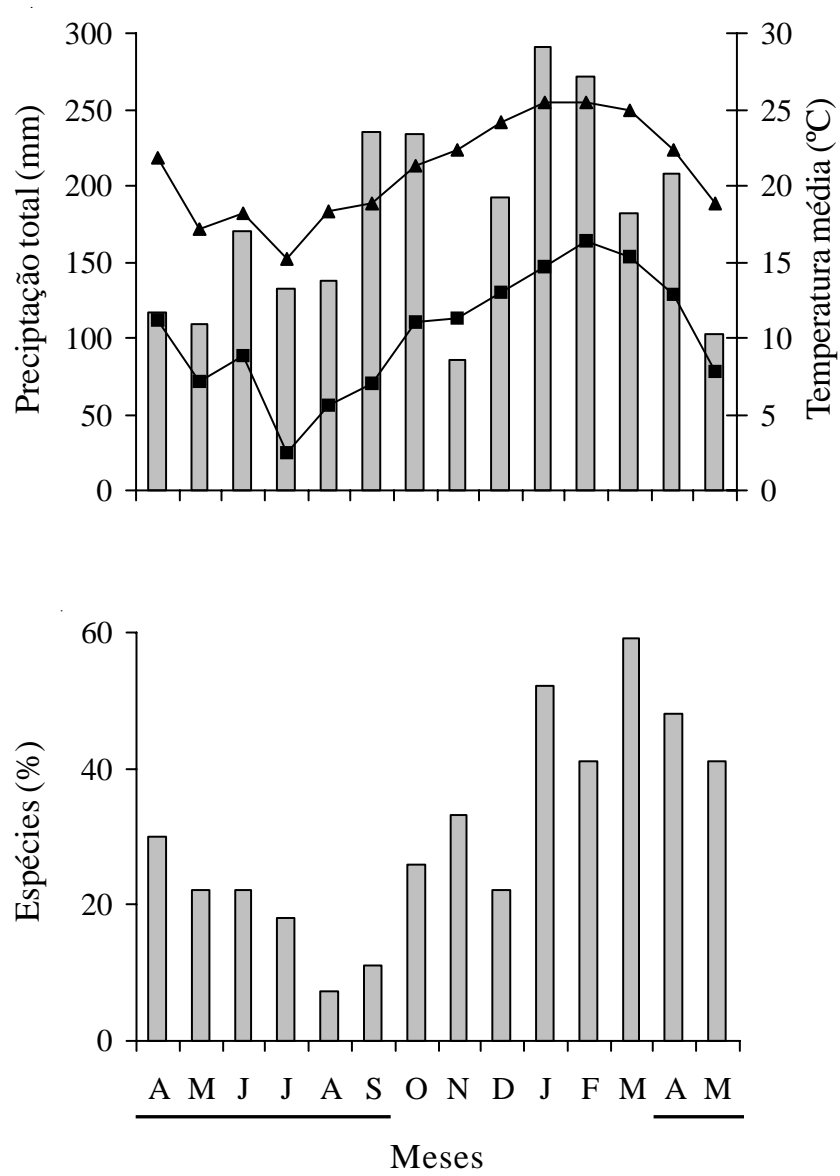

Figura 2. A. Precipitação total mensal (colunas) e valores mensais médios das temperaturas máximas ( $(\mathbf{\Delta})$ e mínimas ( $(\boldsymbol{\square})$ entre abril/2000 e maio/2001 em Floresta Ombrófila Mista, PNAS, RS. B. Padrão de frutificação de angiospermas zoocóricas em porcentagem de espécies $(n=27)$ no PNAS/ RS. Os meses sublinhados indicam produção simultânea de sementes de Araucaria angustifolia.

Figure 2. A. Monthly total rainfall (bars) and mean monthly values of daily maxima ( $\mathbf{\Delta})$ and minima $(\boldsymbol{\square})$ temperatures between April/2000 and May/2001 in Mixed Ombrophilous Forest in south Brazil. B. Patterns of fruit production of zoochoric angiosperms expressed in percentage of the total number of species $(n=27)$ in the NPAS/RS. Underlined months indicate those months when the production of Araucaria angustifolia seeds occurred. pinhão (média $=10,5 ;$ d.p. $=3,9$ ), embora essa diferença não tenha sido significativa (Teste de Mann-Whitney: $\mathrm{U}=10,5 ; \mathrm{p}=0,08)$. De uma forma geral, a frutificação foi mais intensa (número médio de espécies frutificando $55 \%$ maior) durante a época de maior precipitação e temperatura (outubro-março) (figura 2). O número de espécies em frutificação apresentou correlação positiva e significativa com a temperatura média mensal (correlação de Spearman) no mesmo mês $\left(r_{s}=0,78\right.$; $\mathrm{p}=0,001)$, com um mês $\left(\mathrm{r}_{\mathrm{s}}=0,88 ; \mathrm{p}=0,001\right)$ e com dois meses de defasagem $\left(r_{s}=0,75 ; p<0,02\right)$. O número de espécies em frutificação não apresentou correlação significativa com a precipitação total mensal do mesmo mês $\left(r_{s}=0,18 ; p>0,50\right)$. Porém essa correlação foi significativa com a precipitação do mês anterior $\left(\mathrm{r}_{\mathrm{s}}=0,66 ; \mathrm{p}<0,02\right)$, mas não com dois meses de defasagem $\left(r_{s}=0,42 ; p>0,15\right)$.

\section{Discussão}

Segundo Klein (1984), na Floresta Ombrófila Mista geralmente ocorrem entre 25 e 30 espécies de angiospermas arbóreas. Já Jarenkow \& Baptista (1987) e Watzlawick et al. (2002) registraram, em duas áreas distintas de Floresta Ombrófila Mista, a ocorrência de cerca de 37 espécies de angiospermas arbóreas (incluindo espécies zoocóricas e não-zoocóricas). No presente estudo foram encontradas 27 espécies de angiospermas, representando cinco categorias de hábito (arbusto, arvoreta, árvore, erva e epífita), com um total de 11 espécies considerando arvoretas e árvores. Esse número relativamente baixo de espécies arbóreas pode ser uma consequiência do tamanho do fragmento estudado (25 ha) ou estar relacionado a fatores locais (e.g. tipo de solo, perturbação causada pelo pisoteio do gado, corte seletivo de madeira em períodos anteriores à proteção efetiva da área, entre outros).

Em relação à produção de frutos, os nossos resultados mostraram que apenas duas espécies de angiospermas, Myrceugenia miersiana e Leandra variabilis, foram responsáveis por mais de $90 \%$ da produção total de frutos do fragmento estudado. Isso sugere que essas espécies, juntamente com A. angustifolia, seriam as principais fontes de recurso para animais frugívoros em fragmentos de Floresta Ombrófila Mista. Algumas espécies de Melastomataceae (gêneros Leandra, Miconia e Clidemia, entre outros) possuem alta ocorrência de apomixia (Goldenberg \& Varassin 2001), o que pode, em parte, ser responsável pela alta produção de frutos dos representantes dessa família. 
A coloração chamativa dos frutos zoocóricos tem diferentes funções, tais como atrair a atenção de potenciais dispersores, revelar a localização da planta e sinalizar o estágio de maturação do fruto (Willson et al. 1990). No PNAS foram encontrados, mais freqüentemente, frutos nas cores vermelho e laranja, devido principalmente à abundância de espécies de Myrtaceae e Solanaceae. Frutos com essa coloração são geralmente consumidos por aves oportunistas e há evidências que esses animais possuem um papel importante na recomposição de ambientes através da dispersão de plantas pioneiras (Fleming 1988, Whittaker \& Jones 1994, Gorchov et al. 1995). Frutos zoocóricos ocorreram em todas as categorias de hábito encontradas, com predominância das espécies zoocóricas arbustivas. Os frutos zoocóricos da área estudada apresentaram menor variação de tamanho e, conseqüentemente de forma (relação largura $x$ comprimento), quando comparados com outras espécies em florestas no sul do país (e.g. Mikich \& Silva 2001; para Floresta Estacional Semidecidual no Paraná). Variações no tamanho de frutos e sementes também estão relacionadas a fatores físicos e morfogênicos independentes da dispersão (Herrera 1992). O tamanho do fruto é importante para os consumidores, principalmente quando este vai ser consumido inteiro. Assim, o tamanho do fruto pode limitar o número de espécies frugívoras que conseguem obter alimento de uma determinada espécie de planta. No PNAS, o pequeno tamanho dos frutos encontrados sugere que pequenas aves e roedores sejam os animais que dominam a comunidade de frugívoros na área.

Há indícios de que o fragmento estudado se trata de uma área em regeneração, cuja vegetação foi destruída pelo fogo cerca de 50 anos (A. Backes, dados não publicados). Espécies zoocóricas com frutos de pequeno tamanho e coloração atrativa para as aves têm sido citadas como características de áreas em estado sucessional intermediário (Ridley 1930, van der Pijl 1982, Herrera 1995). Desta forma, a alta porcentagem de espécies zoocóricas com frutos com essas características que encontramos reforça essa suposição de que o fragmento estudado encontra-se em processo de sucessão, sem ainda ter atingido o estádio clímax. Alternativamente, isso poderia ser uma característica intrínseca de FOM, mas até o momento não há estudos publicados em outras áreas de FOM que possam corroborar essas suposições.

Das seis espécies mais abundantes e com o maior período de frutificação na área estudada, cinco $(83,3 \%)$ apresentaram distribuição espacial agregada. Esse tipo de distribuição pode ser explicado por vários fatores, como a germinação preferencial em sítios mais favoráveis ao desenvolvimento, a reprodução vegetativa e a distância de dispersão (Janzen 1970). As espécies vegetais estudadas apresentaram frutos e sementes de pequeno tamanho com provável dispersão por aves, o que não justifica a distribuição encontrada. No entanto, pelas observações de campo, o tipo de distribuição espacial encontrado para Leandra variabilis pode estar relacionado, pelo menos em parte, ao seu crescimento clonal (G. Paise, dados não publicados). A reprodução vegetativa é uma estratégia reprodutiva evolutivamente antiga e muito difundida entre diferentes táxons de plantas (Silvertown 1987). Almeida \& Alves (2000) também observaram essa estratégia em indivíduos de Psychotria nuda (Rubiaceae) na região de Floresta Atlântica no sudeste do Brasil. Nossos resultados indicaram distribuição agrupada para L. variabilis e aleatória para L. planifilamentosa na área de estudo. A ocorrência de reprodução sexuada e vegetativa em ambas espécies do gênero Leandra sugere que uma ou outra estratégia prevalece, dependendo das condições ambientais, podendo afetar os padrões de distribuição espacial.

A fenologia de espécies de plantas tem sido relativamente pouco estudada nas florestas brasileiras e a maioria dos trabalhos são recentes (e.g. Morellato \& Leitão Filho 1990, Marinho Filho 1991, Morellato \& Leitão Filho 1992, Alencar 1994, Morellato \& Leitão Filho 1996, Dalponte \& Lima 1999, Ferraz et al. 1999, Almeida \& Alves 2000, Morellato et al. 2000, Talora \& Morellato 2000, Mikich \& Silva 2001, Marques et al. 2004). Alencar (1994) sugeriu que os padrões fenológicos seriam mais afetados pelas características intrínsecas às espécies (genéticas, fisiológicas, reprodutivas) e pelos fatores ecológicos (polinização, predação, competição) do que somente pela influência das variáveis climáticas. Alencar (1994), Ferraz et al. (1999) e Mikich \& Silva (2001) encontraram, em diferentes áreas de estudo, padrões sazonais no período de frutificação da maioria das espécies zoocóricas, enquanto Morellato \& Leitão Filho (1992, 1996) encontraram padrões não sazonais para espécies zoocóricas de floresta semidecidual. Almeida \& Alves (2000), Morellato et al. (2000) e Talora \& Morellato (2000) conduziram estudos em áreas de Floresta Pluvial Atlântica (ambientes fracamente sazonais) e encontraram, para a maioria das espécies zoocóricas, padrões de frutificação contínuos ao longo do ano. No entanto, os dados obtidos no presente estudo (conduzido em uma região mais austral), indicam um padrão distinto, 
com uma acentuada sazonalidade nos padrões de frutificação das espécies.

No único estudo publicado sobre padrões fenológicos em Floresta Ombrófila Mista, Marques et al. (2004) afirmam que a área que eles estudaram, no Paraná, segue o mesmo padrão geral para as florestas tropicais, de pouca variação na produção de frutos ao longo do ano. Eles detectaram também correlações relativamente baixas entre produção de frutos e variáveis climáticas (Marques et al. 2004). Em contraste com esses padrões, os resultados encontrados no presente estudo indicaram que os picos de disponibilidade de frutos ocorrem nos meses de maior precipitação e temperatura (verão), com correlações significativas entre frutificação e a temperatura e a precipitação no mês anterior. As discrepâncias entre os resultados de Marques et al. (2004) e os resultados aqui obtidos podem ser devidos a uma maior sazonalidade climática decorrente das diferenças latitudinais entre os dois estudos.

Estudos conduzidos por Frankie et al. (1974), Hilty (1980), Koptur et al. (1988) e Morellato \& Leitão Filho (1990) indicaram que, em ambientes notadamente sazonais, os fatores ambientais devem ter maior influência sobre as fenofases do que em ambientes pouco sazonais. De acordo com Morellato \& Leitão Filho (1990), em São Paulo, as espécies de floresta semidecídua que possuem frutos carnosos geralmente apresentam produção de frutos na estação de maior precipitação (estação úmida). À medida que nos afastamos do equador, os padrões fenológicos demonstram estar mais influenciados pela temperatura e pelo comprimento do dia do que pela precipitação (Rivera \& Borchert 2001). De modo geral, as espécies analisadas no presente estudo corroboraram este padrão. Inclusive, devido ao inverno muito intenso (com geadas e baixas temperaturas, abaixo de $10{ }^{\circ} \mathrm{C}$ ), algumas plantas passam por períodos de injúria, podendo haver até a perda de folhas, como descrito por Longman \& Jeník (1987). Foster (1982) sugere que a estação em que a planta frutifica está fortemente relacionada com a estação que apresenta condições mais favoráveis para a germinação, não sendo, no entanto, invariavelmente determinada por essa última. No verão, quando ocorre o maior número de espécies frutificando ou a maior produção de frutos, há um aumento na precipitação e, principalmente, nas temperaturas médias, o que pode favorecer a germinação e estabelecimento de novas plântulas. Morellato (1992) observou que, na Serra do Japi, a maior abundância temporária de nutrientes pode ser um fator importante na determinação da época de frutificação de muitas espécies. Na FOM, no verão, há provavelmente um aumento na decomposição da serapilheira, igualmente causado pelo aumento de temperatura e pluviosidade (A. Backes, dados não publicados). Com isso, os nutrientes para a vegetação são disponibilizados e há potenciais vantagens para as plantas frutificarem nessa época.

O período de produção de pinhão (sementes de Araucaria angustifolia) ocorreu em uma época em que havia um menor número de espécies de angiospermas produzindo frutos zoocóricos. Embora essa diferença não tenha sido significativa, ela pode ser biologicamente relevante. O pico de produção de pinhões na área estudada foi entre maio e agosto, período com o menor número médio de angiospermas frutificando e com ausência de árvores em fruto. Essa não sobreposição entre eventos pode favorecer a ocorrência de animais frugívoros, possibilitando uma oferta mais constante de recursos durante todo o ano. Desta forma, a araucária seria uma espécie importante como fonte de recursos para pequenos animais, consumidores de frutos e sementes, em uma época com menor oferta de frutos em geral.

Agradecimentos - Ao Herbarium Anchieta, ao Setor de Botânica da Unisinos em especial a A. Backes pelas conversas e esclarecimentos e a J. Larocca, C.A. Mondin e M. Sobral pelo auxílio na identificação das espécies vegetais. Aos bolsistas e estagiários do Laboratório de Ecologia de Mamíferos - Unisinos que auxiliaram no trabalho de campo e A.C. Alves auxiliou na análise dos frutos. V.B. Ziparro revisou uma versão inicial deste manuscrito e aos revisores anônimos pelas sugestões. Este trabalho recebeu apoio financeiro da Unisinos e Fapergs. Durante a execução deste estudo EMV recebeu bolsa de produtividade do CNPq (Proc. 350330/2002-6).

\section{Referências bibliográficas}

ALENCAR, J.C. 1994. Fenologia de cinco espécies arbóreas tropicais de Sapotaceae, correlacionada a variáveis climáticas na Reserva Ducke, Manaus, AM. Acta Amazonica 24:161-182.

ALMEIDA, E.M. \& ALVES, M.A.S. 2000. Fenologia de Psychotria nuda e P. brasiliensis (Rubiaceae) em uma área de Floresta Atlântica no sudeste do Brasil. Acta Botanica Brasilica 14:335-346.

BACKES, A. 1999. Condicionamento climático e distribuição geográfica de Araucaria angustifolia (Bertol.) Kuntze no Brasil - II. Pesquisas, Série Botânica 49:31-51.

BACKES, A. \& NARDINO, M. 2003. Árvores, arbustos e algumas lianas nativas no Rio Grande do Sul. Editora Unisinos, São Leopoldo. 
BACKES, A., FERNANDEZ, A.V. \& ZENI, A.J. 2000. Produção de folhedo em uma floresta com Araucaria angustifolia no sul do Brasil. Pesquisas, Série Botânica 50:97-117.

BARROSO, G.M., MORIM, M.P., PEIXOTO, A.L. \& ICHASO, C.L.F. 1999. Frutos e sementes: morfologia aplicada à sistemática de dicotiledôneas. Universidade Federal de Viçosa, Viçosa.

BORDIGNON, M. \& MONTEIRO FILHO, E. 1999. Seasonal food resource of the Squirrel Sciurus ingrami in a secondary Araucaria Forest in southern Brazil. Studies of Neotropical Fauna and Environment 34:137-140.

CADEMARTORI, C.V. 1994. Flutuações na abundância de roedores (Cricetidae, Sigmodontinae) ocorrentes em duas áreas de Floresta Ombrófila Mista Montana, Rio Grande do Sul, Brasil. Dissertação de mestrado, Pontifícia Universidade Católica do Rio Grande do Sul, Porto Alegre.

CADEMARTORI, C.V., MARQUES, R.V., PACHECO, S.M., BAPTISTA, L.R.M. \& GARCIA, M. 2002. Roedores ocorrentes em Floresta Ombrófila Mista (São Francisco de Paula, Rio Grande do Sul) e a caracterização de seu habitat. Comunicação do Museu de Ciências Tecnológicas PUCRS, Série Zoologia 15:61-86.

CARVALHO, P.E.R. 1994. Espécies Florestais Brasileiras: recomendações silviculturais, potencialidades e uso da madeira. Empresa Brasileira de Pesquisa Agropecuária, Centro Nacional de Pesquisa de Florestas. EmbrapaCNPF, Colombo.

CRONQUIST, A. 1988. The evolution and classification of flowering plants. New York Botanical Garden, New York.

DALPONTE, J.C. \& LIMA, E.S. 1999. Disponibilidade de frutos e a dieta de Lycalopex vetulus (CarnivoraCanidae) em um cerrado de Mato Grosso, Brasil. Revista Brasileira de Botânica 22:625-332.

FERRAZ, D.K., ARTES, R., MANTOVANI, W. \& MAGALHÃES, L.M. 1999. Fenologia de árvores em fragmento de mata em São Paulo, SP. Revista Brasileira de Biologia 59:305-317.

FERNANDES, A. \& BEZERRA, P. 1990. Estudo fitogeográfico do Brasil. Stylus Publicação, Fortaleza.

FLEMING, T.H. 1988. The short-tailed fruit bat: a study in plant-animal interactions. University of Chicago Press, Chicago.

FOSTER, R.B. 1982. The seasonal rhythm of fruiting on Barro Colorado Island. In The ecology of a tropical forest. (E.G. Leigh Jr., A.S. Rand \& D.M. Windsor, eds.). Smithsonian Institution Press, Washington, p.151-172.

FOURNIER, L.A. 1974. Un método cuantitativo para la medición de características fenológicas en árboles. Turrialba 24:422-423.

FRANKIE, G.W., BAKER, H.G. \& OPLER, P.A. 1974. Comparative phenological studies of trees in tropical lowland wet and dry forest sites of Costa Rica. Journal of Ecology 62:881-913.
GOLDENBERG, R. \& VARASSIN, I.G. 2001. Sistemas reprodutivos de espécies de Melastomataceae da Serra do Japi, Jundiaí, São Paulo, Brasil. Revista Brasileira de Botânica 24:283-288.

GORCHOV, D.L., CORNEJO, F., ASCORRA, C.F. \& JARAMILLO, M. 1995. Dietary overlap between frugivorous birds and bats in the Peruvian Amazon. Oikos 74:235-250.

HERRERA, C.M. 1992. Interespecific variation in fruit shape: allometry, phylogeny and adaptation to dispersal agents. Ecology 73:1832-1841.

HERRERA, C.M. 1995. Plant-vertebrate seed dispersal systems in the Mediterranean: ecological, evolutionary and historical determinants. Annual Review of Ecology and Systematics 26:705-727.

HILTY, S.L. 1980. Flowering and fruiting periodicity in a premontane rain forest in pacific Colombia. Biotropica 12:292-306.

HOWE, H.F. 1980. Monkey dispersal and waste of a tropical fruit. Ecology 61:944-959.

HOWE, H.F. 1986. Seed dispersal by fruiting-eating birds and mammals. In Seed dispersal (D.R. Murray, ed). Academic Press, Sydney, p.123-183.

HOWE, H.F. \& SMALLWOOD, J. 1982. Ecology of seed dispersal. Annual Review of Ecology and Systematics 13:201-28.

HOWE, H.F., SCHUPP, E. \& WESTLEY, L.C. 1985. Early consequences of seed dispersal for a neotropical tree Virola surinamensis. Ecology 66:781-791.

JANZEN, D.H. 1970. Herbivores and the number of trees in tropical forests. American Naturalist 104:501-528.

JARENKOW, J.A. \& BAPTISTA, L.R.M. 1987. Composição florística e estrutura da mata com araucária na Estação Ecológica de Aracuri, Esmeralda, Rio Grande do Sul. Napaea 3:9-18.

KLEIN, R.M. 1984. Aspectos dinâmicos da vegetação do sul do Brasil. Sellowia 36:5-54.

KÖPPEN, W. 1948. Climatologia. Ed. Fondo Cultura Económica, Ciudad de México.

KOPTUR, S., HABER, W.A., FRANKIE, G.W. \& BAKER, H.G. 1988. Phenological studies of shrub and treelet species in tropical cloud forests of Costa Rica. Journal of Tropical Ecology 4:323-346.

KREBS, C.J. 1989. Ecological methodology. Harper \& How, New York.

LEITE, P. \& KLEIN, R.M. 1990. Vegetação. In Geografia do Brasil: Região Sul. Instituto Brasileiro de Geografia e Estatística, Rio de Janeiro, v.2, p.113-150.

LONGMAN, K.A. \& JENÍK, J. 1987. Tropical forest and its environment. Longman Scientific \& Technical, New York.

MANTOVANI, A., MORELLATO, L.P.C. \& REIS, M.S. 2004. Fenologia reprodutiva e produção de sementes em Araucaria angustifolia (Bert.) O. Kuntze. Revista Brasileira de Botânica 27:787-796. 
MARINHO FILHO, J.S. 1991. The coexistence of two frugivorous bat species and the phenology of their food plants in Brazil. Journal of Tropical Ecology 7:59-67.

MARQUES, C.M., ROPER, J.J. \& SALVAGGIO, A.P.B. 2004. Phenological patterns among plant life-forms in a subtropical forest in southern Brazil. Plant Ecology 173:203-213.

MIKICH, S.B. \& SILVA, S.M. 2001. Composição florística e fenologia das espécies zoocóricas de remanescentes de floresta estacional semidecidual no Centro-oeste do Paraná, Brasil. Acta Botanica Brasilica 15:89-113.

MORELLATO, L.P.C. 1992. Sazonalidade e dinâmica de ecossistemas florestais na Serra do Japi. In História natural da Serra do Japi: ecologia e preservação de uma área florestal no Sudeste do Brasil. (L.P.C. Morellato, org.). Editora da Unicamp/Fapesp, Campinas, p.98-110.

MORELLATO, L.P.C. \& LEITÃO FILHO, H.F. 1990. Estratégias fenológicas de espécies arbóreas em floresta mesófila na Serra do Japi, Jundiaí, São Paulo. Revista Brasileira de Biologia 50:163-173.

MORELLATO, L.P.C. \& LEITÃO FILHO, H.F. 1992. Padrões de frutificação e dispersão na Serra do Japi. In História natural da Serra do Japi: ecologia e preservação de uma área florestal no Sudeste do Brasil. (L.P.C. Morellato, org.). Editora da Unicamp/Fapesp, Campinas, São Paulo, p.112-140.

MORELLATO, L.P.C. \& LEITÃO FILHO, H.F. 1996. Reproductive phenology of climbers in a Southeastern Brazilian forest. Biotropica 28:180-181.

MORELLATO, L.P.C., RODRIGUES, R.R., LEITÃO FILHO, H.F. \& JOLY, C.A. 1989. Estudo comparativo da fenologia de espécies arbóreas de floresta de altitude e floresta mesófila semidecídua na Serra do Japi, Jundiaí, São Paulo. Revista Brasileira de Botânica 12:85-98.

MORELLATO, L.P.C., TALORA, D.C., TAKAHASI, A., BENCKE, C.C., ROMERA, E.C. \& ZIPARRO, V.B. 2000. Phenology of atlantic rain forest trees: a comparative study. Biotropica 32:811-823.

RAMBO, B. 1953. História da flora do Planalto Riograndense. Anais Botânicos do Herbário Barbosa Rodrigues 5:185-232.
REITZ, R. \& KLEIN, R.M. 1966. Araucariáceas. In Flora Ilustrada Catarinense (R. Reitz, ed.). Herbário Barbosa Rodrigues, Itajaí, Parte I, p.1-62.

REITZ, R., KLEIN, R.M. \& REIS, A. 1988. Projeto Madeira do Rio Grande do Sul. Secretaria de Agricultura e Abastecimento, Sudesul, Porto Alegre.

RIDLEY, H.N. 1930. The dispersal of plants throughout the world. Reeve, Ashford.

RIVERA, G. \& BORCHERT, R. 2001. Induction of flowering in tropical trees by a $30-\mathrm{min}$ reduction in photoperiod: evidence from field observations and herbarium specimens. Tree Physiology 21:201-212.

RIZZINI, C.T. 1992. Tratado de fitogeografia do Brasil: aspectos ecológicos, sociológicos e florísticos. $2^{\mathrm{a}} \mathrm{ed}$. Âmbito Cultural, Rio de Janeiro.

SILVERTOWN, J. 1987. Introduction to plant population ecology. $2^{\text {nd }}$ ed. Longman Scientific \& Technical, New York.

TALORA, D.C. \& MORELLATO, L.P.C. 2000. Fenologia de espécies arbóreas em floresta de planície litorânea do sudeste do Brasil. Revista Brasileira de Botânica 23:13-26.

VAN DER PIJL, L. 1982. The principles of dispersal in higher plants. $3^{\text {rd }}$ ed. Springer-Verlag, Berlin.

VIEIRA, E.M. \& IZAR, P. 1999. Interactions between aroids and arboreal mammals in the Brazilian Atlantic rainforest. Plant Ecology 145:75-82.

WATZLAWICK, L.M., RONDON NETO, R.M., CALDEIRA, M.V.W. \& SCHOENINGER, E.R. 2002. Aspectos florístico e estrutural de um fragmento de floresta com araucária utilizado para o pastoreio de bovinos. Ciência \& Natura 24:63-76.

WHITTAKER, R.J. \& JONES, S.H. 1994. The role of frugivorous bats and birds in the rebuilding of a tropical forest ecosystem, Krakatau, Indonesia. Journal of Biogeography 21:245-258.

WILLSON, M.F., GRAFF, D.A. \& WHELAN, C.J. 1990. Color preferences of frugivorous birds in relation to the colors of fleshy fruits. The Condor 92:545-555.

WILKINSON, L. 1998. Systat, the system for statistics. Systat Inc., Evanston.

ZAR, J.H. 1996. Biostatistical analysis. $3^{\text {rd }}$ ed. Prentice-Hall, New Jersey. 